\title{
A. Parker* \\ Confirming the lack of any sea level acceleration around the Australian coastline
}

\begin{abstract}
Even if sea levels fluctuate with decadal and multi decadal periodicities about a linear trend without any positive acceleration component, many papers continue to compare different compilations of tide gauges of different length and different locations or assemble selected tide gauges of selected length and location to prove that sea levels are accelerating in Australia and elsewhere in the world when they are not.

It is proposed here a novel procedure fitting the tide gauge data with linear and multiple sinus functions that also iteratively completes the gaps in the recorded data with subsequent guess of the fitted function. It is shown that the tide gauges of Sydney and Fremantle, the only two exceeding a century in Australia, are acceleration free and naturally oscillating with different periodicities, phases and amplitudes for the Indian and Pacific Ocean locations. It is also shown that tide gauge records of length smaller than 60 years have rates of rise differing considerably from the legitimate long term trends, and these values may change significantly from an update to the other because of the natural oscillations. It is definitively assessed that tide gauge records of length about 20 years, with a starting point at the time of a valley of one peak and valley multi decadal oscillation for the specific of the most part of the coastline of Australia, return completely unrealistic values for the rate of rise of sea levels. The only reliable assessment of sea level trends along the coastline of Australia continues to be the determination of the rate of rise by linear fitting of all the available data in all the available station, providing the total numbers of years recorded exceed at least 25 years to make the population significant, as historically done by the National Tide Centre of the Australian Bureau of Meteorology prior of the censored 2009 survey. While some changes are expected in the individual values of the rate of rise from one of these surveys to the other, and the introduction of new stations satisfying the 25 years requirement could also affect the average tide gauge result, these sea level surveys conducted without cherry picking the station and the time window generally provide about same average sea level rates of rise survey after survey.
\end{abstract}

*Corresponding Author: A. Parker: RMIT University, Bundoora, VIC 3083, Australia, E-mail: Albert.parker@rmit.edu.au

\section{The sea level rise around Australia is not accelerating}

The recent paper by the group of the Coordinating Lead Author of the latest IPCC WGI Fifth Assessment Report Chapter 13: Sea Level Change [1], famous for the continuously positively accelerating reconstructions of sea levels following the anthropogenic carbon dioxide emissions but not the temperatures as peer reviewed in $[2,3]$, reproposes the issue of misrepresenting the sea level rate of rise around Australia by cherry-picking the sea level records where and when more convenient to support claims of present rates of rise much higher than in the past.

As already written in [4-6], the sea levels oscillate with periodicities up to a quasi-60 years periodicity detected, possibly because the temperatures oscillate with this same periodicity [7-9], about a longer term trend. While this longer term trend for temperatures is possibly also a longer term oscillation [4-6], for the sea levels is a linear function of time. While other shorter periodicities are also relevant for the temperatures and sea levels, it does not make too much sense to focus on records of sea levels shorter than 60 years to infer the sea level rate of rise, and it does make even less sense to focus only on the records started in a recent valley of a multi decadal oscillations only 20 years away as done in the Australian and South Pacific sea level monitoring projects and partially done in the paper [1] that support the use of the 1993- 2011 time window.

Before the censored survey of 2009, the National Tide Centre of the Australian Bureau of Meteorology was producing annual surveys to provide a synopsis of the annual mean sea level trends in longer-term relative sea level records. In the 2009 survey, there were 39 Australian locations where relative sea levels were measured for at least 25 years. The average length of these records was 42 years. Two new locations (Wallaroo and Cape Ferguson) qualified in 2009 as long-term stations with more than 25 years of data. The average trend from all the 39 stations was $0.9 \mathrm{~mm} /$ year with a standard deviation of $1.9 \mathrm{~mm}$ /year. Because some of the stations were exhibiting unrealistic trends due to undocumented datum shifts, a more realistic average trend was obtained from 29 stations within 1 $\mathrm{mm}$ /year standard deviation of the mean as $1.4 \mathrm{~mm}$ /year 
with a standard deviation of $0.7 \mathrm{~mm} /$ year. The geographical pattern of relative sea level trends around the Australian coastline is fairly uniform in general. Annual mean sea levels around the Australian coastline are strongly correlated with the El Niño - Southern Oscillation (ENSO) signal. Annual mean sea levels generally fluctuate in accordance with the Southern Oscillation Index (SOI) with periods of around 20 years.

This approach was everything but perfect. First of all, the standard deviation of the mean is simply a measure of the linear fitting error, i.e. how close the measured monthly sea levels are to the linear fit. Being the sea levels oscillating about the linear trend with many different periodicities, and being the longer periodicities much larger than the 25 years, the use of the standard deviation from the linear fitting as a parameter for the quality of the record is questionable. Second, the presence of longer periodicities for the fluctuations was not recognised. Finally, quality issues as missed data in the records or other biasing factors as change of instrument or construction works close to the tide gauge location were not accounted for in the study. However, these surveys were replaced by the selective focus on just a few selected tide gauges all started from a known valley of the peak and valley multi decadal oscillations of the Australian Baseline Sea Level Monitoring project everything but more reliable.

In tide gauge time series, the dependent y-values, the monthly average sea levels, are a function of the independent $\mathrm{x}$-values, the time in years. The classic approach to analyse sea level data is to use a linear fit:

$$
y=y_{0}+a \cdot x
$$

In this equation, $\mathrm{y}_{0}$ and a are constants to compute the average sea level rise over the period of observation. The length of the record strongly affect the computation of the sea level rate of rise SLR=a. This can be easily realised when long records are available, but it is impossible to understand if only few years of data are available.

From a measured distribution $\mathrm{x}_{i}, \mathrm{y}_{i}$ for $\mathrm{i}=1, \mathrm{~N}$ it is possible to estimate many $\mathrm{SLR}_{j, k}$ by linearly fitting all the data over the time windows $\mathrm{x}_{j}$ to $\mathrm{x}_{k}$. At a certain time $\mathrm{x}_{k}, \mathrm{x}_{j}$ is taken as $\left(\mathrm{x}_{k}-20\right),\left(\mathrm{x}_{k}-30\right)$ or $\left(\mathrm{x}_{k}-60\right)$ years respectively when computing the $\mathrm{SLR}_{20}, \mathrm{SLR}_{30}$ and $\mathrm{SLR}_{60}$, or as $\mathrm{x}_{1}$ when computing the $\mathrm{SLR}_{A}$ over all the years. As shown in Figure 2 of [6], the sea level rates of rise computed at any time by using 20, 30, 60 years or all the available data are largely oscillating in long term tide gauges as Sydney and Fremantle, the only two centenary tide gauges of Australia, as a result of the decadal and multi-decadal oscillations. The $\mathrm{SLR}_{20}$ and SLR 30 oscillate significantly, and the $\mathrm{SLR}_{60}$ also oscillate even if much less. Even the $\mathrm{SLR}_{A}$ has an os- cillating behaviour, and if the time rate of change of the $\mathrm{SLR}_{A}$ is considered the instantaneous sea level acceleration SLA, this parameter fluctuates about zero. Records of short length do not permit to assess any trend in sea level rise. As shown in Figure 4 of [6], both the tide gauges of Fremantle and Sydney have monthly mean sea levels results that may be fitted very well with a line plus several cosines of periodicities all smaller than the record length, and if the monthly mean sea levels perfectly oscillate about a linear trend, this is another proof that there is then no acceleration in the record to couple with the SLA.

Our latest estimation of the rates of rise of sea levels was Table 1 of [5] was assembled by using same approach of the censored 2009 survey of the National Tide Centre of the Australian Bureau of Meteorology for consistency and compared to the misleading table of sea level rises of the Australian Baseline Sea Level Monitoring project. With a longer average record length, the oldest 39 stations of the Table 1 of [5] provided an average sea level rate of rise of 0.9 $\mathrm{mm}$ /year i.e. exactly same value of the prior censored survey. In the work [5], the total number of stations of record length exceeding 25 years was at the time 47, and the average sea level rate of rise on the total population was 1.2 $\mathrm{mm} /$ year, but clearly there was no reason to compare this latter value with the 2009 value because of the changed demography.

The latest work [1] uses of three temporal windows (1900-2011, 1966-2011 and 1993-2011) without having for the same time windows the same tide gauges because the demography of the population changes dramatically from one time window to the other, and uses a time window of 19 years definitively too short. The proposal of the 3 different average rates of rise computed with time windows weighting more and more the recent times provides the very wrong impression that the rates of rise of sea levels are presently accelerating when it is not. The "weighted average rates of sea level rise of 1.4, 1.7 and $4.6 \mathrm{~mm} /$ year over the time periods beginning in 1900, 1966 and 1993" proposed in [1] are misleading and lack of any scientific evidence similarly to the always accelerating reconstructed global sea levels of [2] reaching their maximum values after more than a decade of lack of any warming [3] where it should be well accepted that only positive temperature gradients may produce sea level accelerations [13]. Fremantle and Sydney are the only two tide gauges with data from the year 1900. Therefore, the average tide gauge 1900 - 2011 is made of a population of two. Many more stations are open since 1966, and because every tide gauge has his own unicity with rates of rises differing from location to location even if oscillations are similar in same geographical areas, the average rate of rise 1966 - 2011 may be 
larger or smaller than the average rate of rise 1900 - 2011 simple because of the new tide gauges of larger or smaller rates of rise without proving anything in terms of acceleration. The 45 years range is also generally short to compute a meaningful sea level rate of rise being the sea levels globally affected by quasi 60 years oscillation and longer, but the need to have a significant population suggests lowering the minimum number of years bar. Only Fremantle, Fort Denison (Sydney), Port Pirie and Port Adelaide (Outer Harbour) satisfy indeed the minimum 60 years requirement. Finally, all the stations are open since 1993, but the less than 20 years of recording are absolutely not worth of any consideration. It does not make any sense to consider 19 years short records incidentally starting from a recent valley of the peak and valley of a multi-decadal oscillation to infer sea level rates of rise unrealistically large [5-7].

If the needs to consider records long enough to compute a meaningful trend has been already commented, the other mistake of changing demography time after time also need attention. It is unfortunately a common practice of many reconstructions and compilation to change dramatically the demography of the tide gauges to infer globally accelerating trends from tide gauges all individually not accelerating. If we do consider the worldwide tide gauges included in the PSMSL data base [10], the most part of the oldest tide gauges are located in Northern Europe, where many countries as Finland or Norway or Sweden are subject to isostasy and the rates of rise are small or even negative. Conversely, many more recent tide gauges are located in other areas where the sea levels oscillate much more or the tide gauges are subject to subsidy as in the Pacific atolls. Without accounting for the changed demography, the different weight of Pacific or North European tide gauges of different length over the time may give a false impression of acceleration even if all the stacked time series are not accelerating.

\section{Linear and multiple sinus fittings}

We propose here a further refinement of the methods proposed in [6] to analyse tide gauge records, and we apply these methods to the tide gauge records of Sydney and Fremantle updated December 2012 (data from [10]). We do use a linear fitting coupled to multiple non-linear fittings with sinus to better describe the tide gauge behaviour. The fitting curve is:

$$
y=\left(y_{0}+a \cdot x\right)+\sum_{i=1}^{n}\left[y_{0, i}+A_{i} \cdot \sin ^{b_{j}}\left(\pi \cdot \frac{x-x_{c, i}}{w_{i}}\right)\right]
$$

In this equation, $\mathrm{n}$ is the number of sinus functions and $\mathrm{y}_{0}, \mathrm{a}, \mathrm{y}_{0, i}, \mathrm{~A}_{i}, \mathrm{x}_{c, i}, \mathrm{w}_{i}, \mathrm{~b}_{j}$ are the fitting coefficients. In this work, we limit our attention to the first 8 periodicities.

Sydney has two tide gauge records in Fort Denison, SYDNEY, FORT DENISON (PSMSL ID 65, latitude -33.850, longitude 151.233, Time span of data 1886 - 1993, Completeness (\%) 100) and SYDNEY, FORT DENISON 2 (PSMSL ID 196, latitude -33.855 , longitude 151.226, Time span of data 1914 - 2012, Completeness (\%) 98). The two tide gauges have almost 80 years of successful overlapping and the integration of the two records in a composite record spanning 1886 to 2012 without any gaps does not pose any quality issue.

Fremantle has only one tide gauge, FREMANTLE (PSMSL ID 111, latitude -32.065556, longitude 115.748139, Time span of data 1897 - 2012, Completeness (\%) 92). This tide gauge has significant gaps, and the way the gaps are filled interpolating neighbouring years may change the result of a Fourier analysis of the time series. In the present approach, the parameters of equation (2) are computed first from the measured data, then the gaps in the measured data are filled with the estimation from equation (2) and new parameters of equation (2) are computed. This iterative procedure is the best approach to avoid introducing unrealistic periodicities of oscillations.

Figure 1.a presents the composite tide gauge record 1886 - 2012 for Sydney. The 12 months moving averages are superimposed. The sea level rate of rise in Sydney is apparently constant at about $0.65 \mathrm{~mm}$ /year. Figure 1.b presents the monthly oscillations about the linear trend. Figures 1.c, d present the comparison of the measured monthly sea levels with the values computed with linear and multiple sinus fitting. Over the last 30 years the sea levels have clearly naturally oscillated without any sign of acceleration. Figure 1.e presents the GPS signal of a nearby SONEL station. The nearby GPS indicates a land motion of $-0.89+/-0.65$ $\mathrm{mm} /$ year [11].

Figure 2.a presents the composite tide gauge record 1897 - 2012 for Fremantle. The 12 months moving averages are superimposed. The sea level rate of rise in Fremantle is apparently constant at about $1.65 \mathrm{~mm}$ /year. Figure 2.b presents the monthly oscillations about the linear trend.

Figures 2.c,d present the comparison of the measured monthly sea levels with the values computed with linear and multiple sinus fitting. Over the last 30 years the sea levels have clearly naturally oscillated without any sign of acceleration. Figure 2.e presents the GPS signal of the closest SONEL station. The PERTH GPS indicates a land motion of $-2.99+/-0.34 \mathrm{~mm} /$ year [11]. The GPS signal quality is affected by 4 offsets for material change or malfunction and 1 offset for unknown reason [11]. 


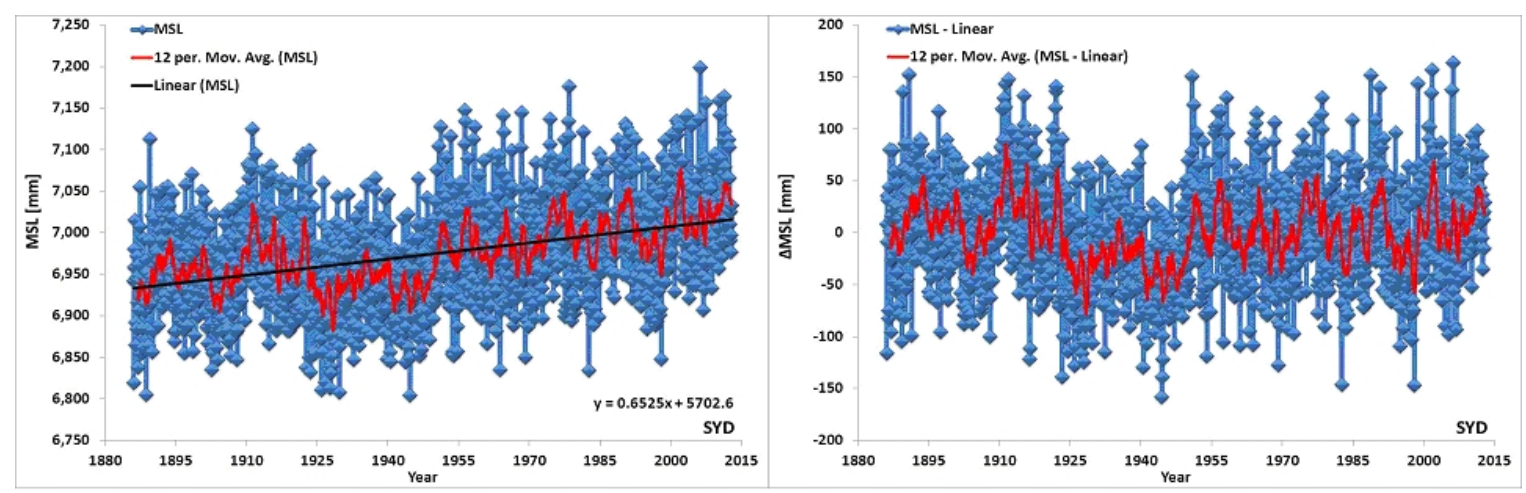

(a)

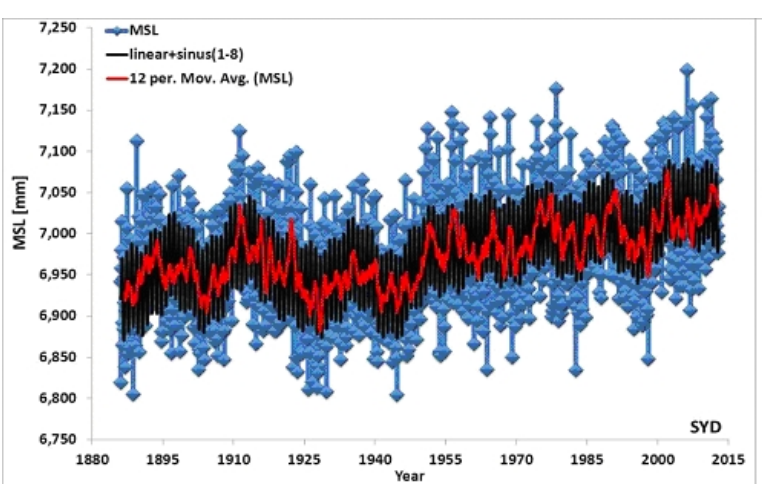

(b)

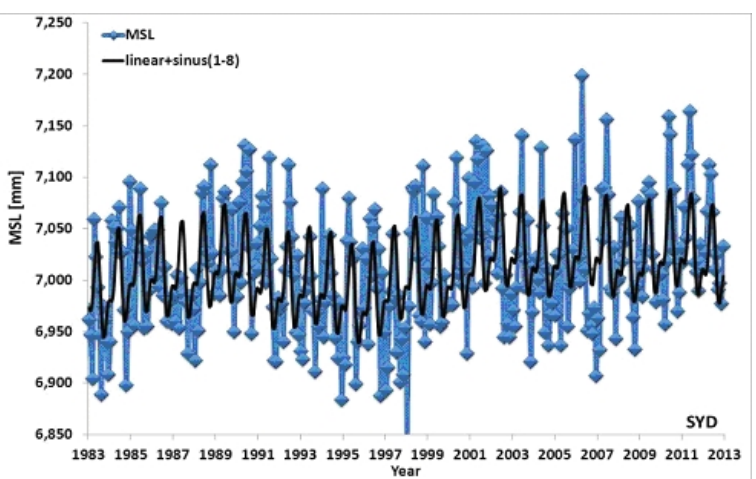

(d)

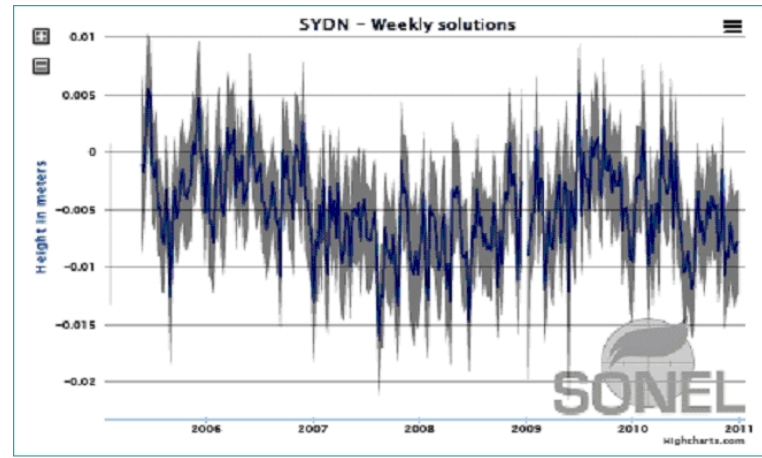

(e)

Fig. 1. Sea levels in Sydney: (a) monthly sea levels; (b) oscillations of monthly sea levels about the linear trend; (c, d) measured monthly sea levels and computed with linear and multiple sinus fitting; (e) land motion in the nearby GPS station.

While we do not support the correction of the tide gauge result by satellite altimetry for multiple reasons, because the GPS is only available since few years, because over these years the GPS had significant vertical inaccuracies, and because what is actually of interest apart from the global warming debate is the relative motion sea to land, the higher SLR of Perth are very likely the result of local ef- fects very likely the vertical motion of the land (but other effects may also be relevant).

The Fourier analyses of the Sydney and Fremantle time series are significantly different. The iterative process to compute the parameters of the sinus fitting in equation (2) may return periodicities differing from the periodogram especially in the longer periodicities because of lack of data and noise. Noise in the measurements and length of 

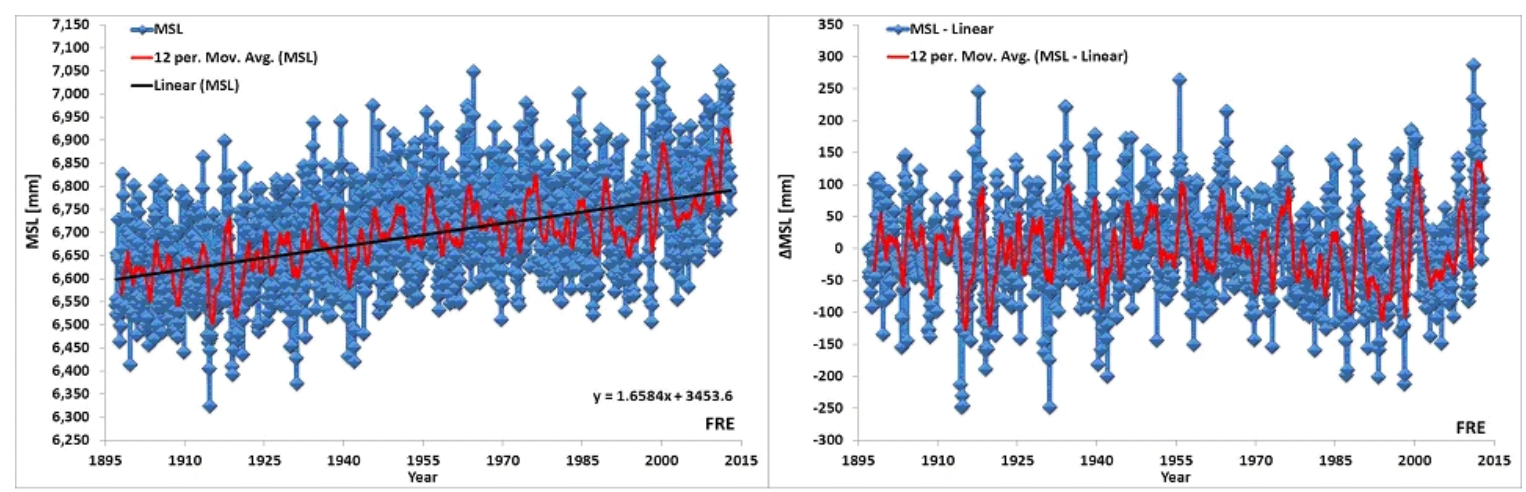

(a)

(b)
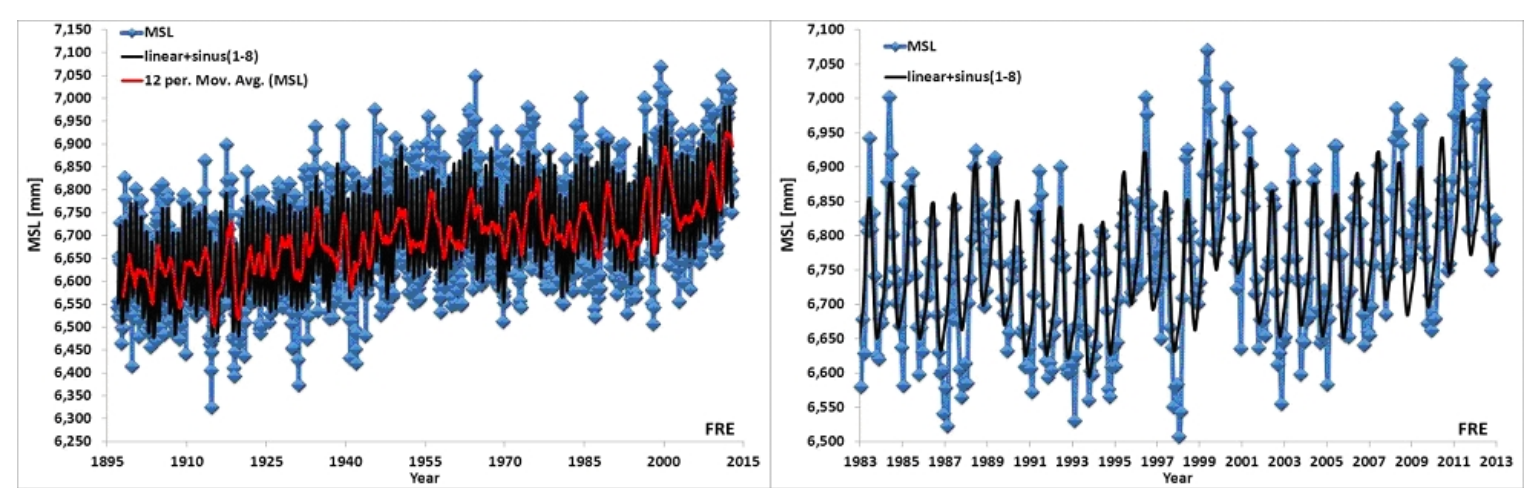

(c)

(d)

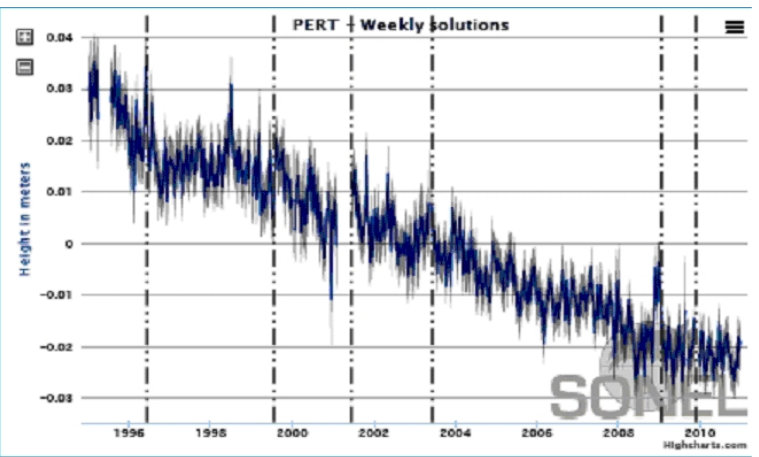

(e)

Fig. 2. Sea levels in Fremantle: (a) monthly sea levels; (b) oscillations of monthly sea levels about the linear trend; (c, d) measured monthly sea levels and computed with linear and multiple sinus fitting; (e) land motion in the Perth GPS station.

the record limit the accuracy of the detection especially of the longer periodicities. The periodicities of the oscillations considered in Sydney are 1, 0.5, 91.28, 22.58, 18.78, 12.94 and 52.04 years. The periodicities of the oscillations considered in Fremantle are 1, 0.5, 5.59, 4.13, 12.46, 3.59, 67.56 and 11.58 years. Apart from the 1 and 0.5 years periodicities, the oscillations are mostly different in phase, am- plitude and period in Fremantle (Indian Ocean) and Sydney (Pacific Ocean).

While a quasi-60 years periodicity was detected in [6] in the first and second harmonics exceeding 1 years of Sydney and Port Pirie, same long term periodicity was not detected in Fremantle and Port Adelaide (Outer Harbour), the only two other tide gauges of Australia exceeding the 60 years length. Existence of a quasi-60 year fluctuation in 

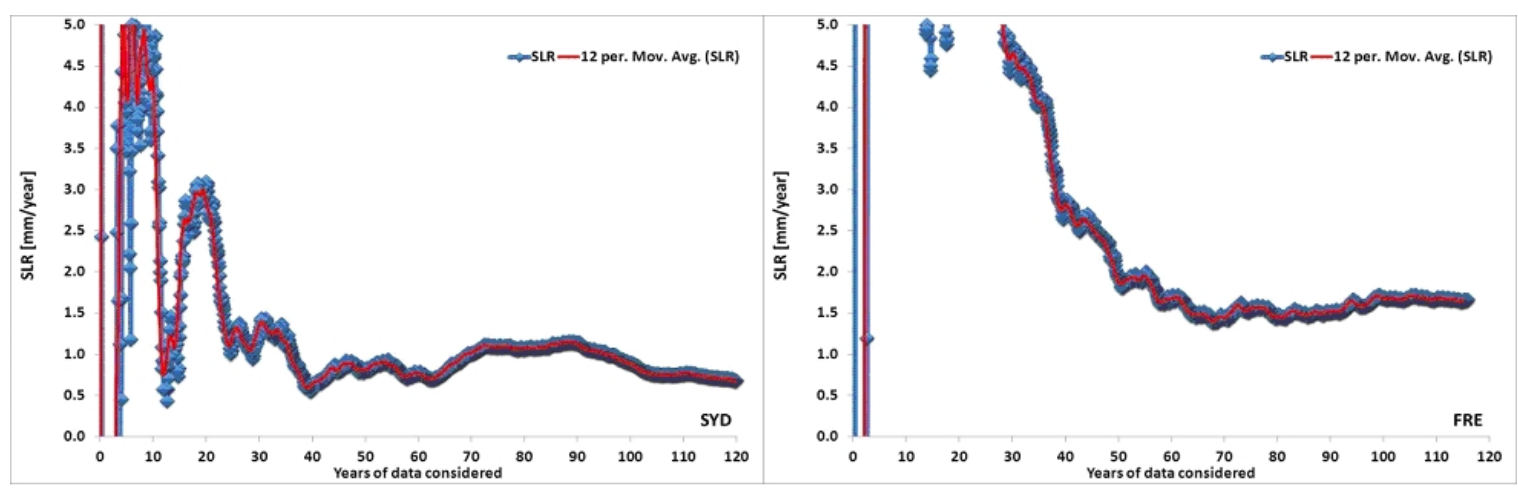

(a)

(b)
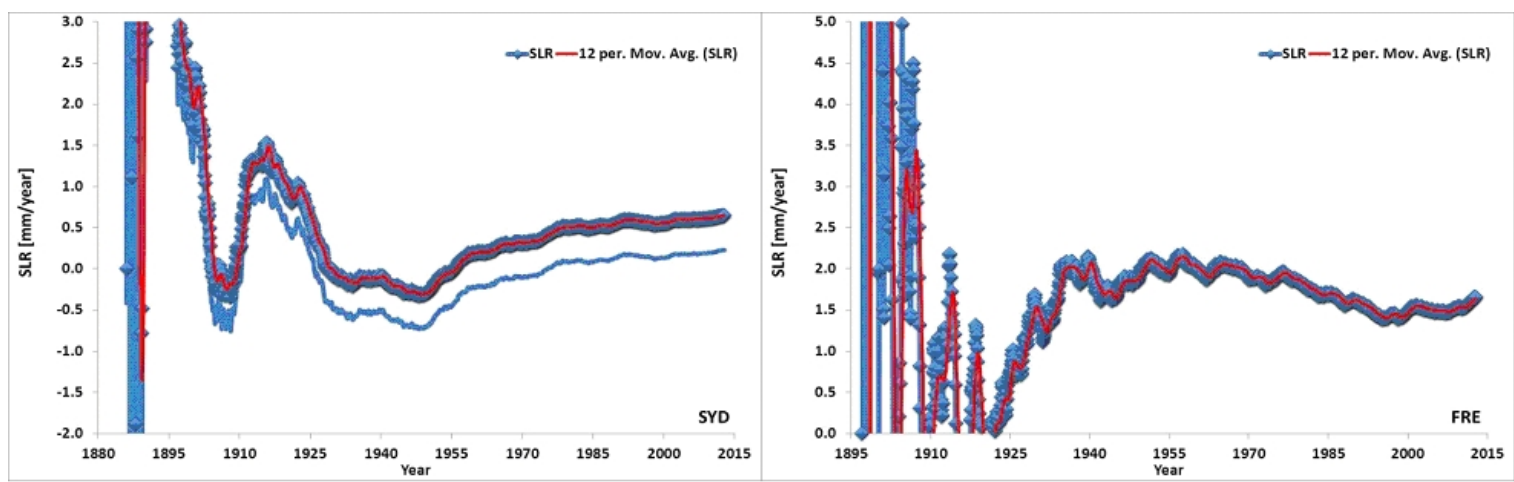

(c)

(d)

Fig. 3. Sea level rate of rise computed by linear fitting in Sydney (a) and Fremantle (b) considering different starts of the record up to the present time and time history of the rate of rise computed at any time by suing all the data available in Sydney (c) and Fremantle (d).

Fremantle and Sydney is found also in [12], confirming the noticeable shift in the phase, however, between the Indian Ocean and South Pacific gauges. Within the limit of two only tide gauges considered to cover the Indian Ocean, the phase of the quasi-60 years oscillation in the Indian Ocean seems similar to that in the Atlantic and Western North Pacific, while that in the South Pacific seems to lag the other basins by about 10 years [12].

Figure 3 finally presents the SLR computed by linear fitting in Sydney and Fremantle considering different starts of the record up to the present time, as well as the $\mathrm{SLR}_{A}$ time histories. These pictures clearly show that in case the records in Sydney and Fremantle could have started 20 years ago in 1993, the SLR in Sydney and Fremantle could have been more than 4 times the legitimate long term value. Similarly, the $4.6 \mathrm{~mm} /$ year of the $1993-$ 2011 time window of [1] is very likely more than 4 times the true long term value.

\section{Conclusions}

Wrongly inferred accelerating sea levels are only the result of selectively filtering the tide gauges to be considered in the compilations and selecting the short time windows that may be supportive of the claim.

All the long term tide gauges worldwide exhibit same not accelerating behaviour and similar pattern of natural oscillations about a linear trend as it is shown for Sydney and Fremantle.

Compilations of tide gauges may be subject to small changes in the average rate of rise because of the natural oscillations in positive and negative. Individual changes may be larger the shorter is the tide gauge record.

Ideally, only stations with more than 60 years of recorded data without gaps should be considered to compute trends. For the specific of Australia, 25 years may be accepted for legacy with past good works.

Being the rate of rise variable worldwide from largely negative to largely positive values for multiple reasons, 
changes in the demography of the stations may produce unrealistic accelerations or decelerations even stacking individually not accelerating records.

The use of tide gauge records of length 20 years and less to infer sea level rise trends in Australia, and more specifically starting about 1993 , should be discouraged producing results very far from legitimate.

The sea level rate of rise around Australia is not accelerating, as not accelerating is the sea level everywhere else, and being the worldwide temperatures unchanged since the beginning of the century there is no reason why they should.

\section{References}

[1] Reed J. Burgette, Christopher S. Watson, John A. Church, Neil J. White, Tregoning P. and Coleman R., "Characterizing and minimizing the effects of noise in tide gauge time series: relative and geocentric sea level rise around Australia”, Geophysical Journal International

[2] Church J. A. and N.J. White, "Sea-level rise from the late $19^{\text {th }}$ to the early $21^{\text {st }}$ Century", Surveys in Geophysics 2011; 32(45):585-602.

[3] Parker A., "Mismatch between acceleration of reconstructed sea levels and gradient of reconstructed temperatures", accepted paper, in press (2013).

[4] Parker A. "Sea level trends at locations of the United States with more than 100 years of recording", Natural Hazards 2013; 65(1):1011-1021.

[5] Parker A. "Oscillations of sea level rise along the Atlantic coast of North America north of Cape Hatteras", Natural Hazards 2013; 65(1):991-997.

[6] Parker A., Saad Saleem M., Lawson M. "Sea-level trend analysis for coastal management", Ocean and Coastal Management 2013; 73:63-81.

[7] Scafetta N., "Discussion on climate oscillations: CMIP5 general circulation models versus a semi-empirical harmonic model based on astronomical cycles", Earth-Science Reviews 2013; 126:321-357.

[8] Scafetta N., "Solar and planetary oscillation control on climate change: hind-cast, forecast and a comparison with the CMIP5 GCMs”, Energy \& Environment 2013; 24(3-4):455-496.

[9] Scafetta N., "Does the Sun work as a nuclear fusion amplifier of planetary tidal forcing?", paper presented At the Space Climate Symposium-5 in Oulu, Finland. June 15-19, 2013. people.duke.edu/ ns2002/pdf/Scafetta-Oulu.pdf.

[10] http://www.psmsl.org/data/obtaining/

[11] http://www.sonel.org/

[12] Chambers D. P., Merrifield M.A., and Nerem R. S., "Is there a 60-year oscillation in global mean sea level?”, Geophysical Research Letters 2012; 39:L18607.

[13] Rahmstorf S., "A semi-empirical approach to projecting future sea-level rise", Science 2007; 315(5810):368-370. 